
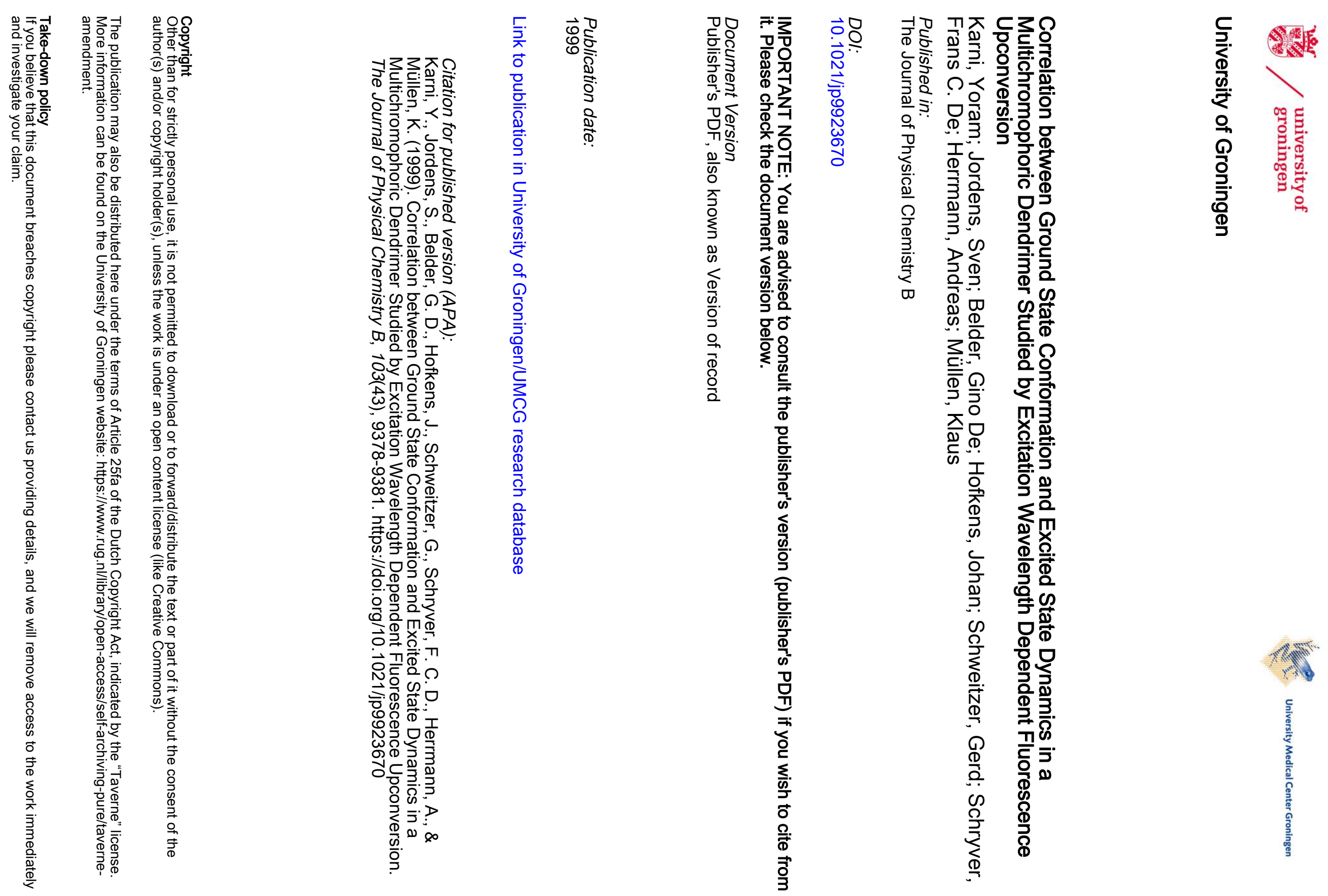


\title{
Correlation between Ground State Conformation and Excited State Dynamics in a Multichromophoric Dendrimer Studied by Excitation Wavelength Dependent Fluorescence Upconversion
}

\author{
Yoram Karni, Sven Jordens, Gino De Belder, Johan Hofkens, Gerd Schweitzer, and \\ Frans C. De Schryver* \\ Department of Chemistry, Katholieke Universiteit Leuven, Celestijnenlaan 200F, 3001 Heverlee, Belgium
}

\author{
Andreas Herrmann and Klaus Miillen \\ Max-Planck-Institut für Polymerforschung, Ackermannweg 10, 55128 Mainz, Germany
}

Received: July 14, 1999; In Final Form: August 22, 1999

\begin{abstract}
A time-resolved fluorescence upconversion study on a polyphenylene dendrimer with 16 peryleneimide chromophores at the rim, in which the excitation wavelength is systematically varied while the detection wavelength is kept constant, is reported. A new setup is described which allows excitation at wavelengths as short as $320 \mathrm{~nm}$ and throughout the visible range up to $900 \mathrm{~nm}$ with a system prompt response on the order of $300 \mathrm{fs}$. In addition to results reported previously $\mathrm{y}^{13}$ which were obtained at shorter wavelengths indicating the initial formation of a locally excited state which then evolves further into a locally excited state on a 5 ps time scale, the newly obtained data at longer wavelength excitation show the existence of a ground state interaction leading under these conditions of excitation predominantly to a directly formed complex.
\end{abstract}

\section{Introduction}

Dendrimers are highly branched macromolecular systems whose structure can be defined on a molecular level ${ }^{1,2}$ and as such have been attracting a lot of attention not only from the synthetic point of view ${ }^{3}$ but also from the point of view of their physical and chemical properties. ${ }^{4-8}$

Recently, dendrimers consisting of a polyphenylene core and decorated with peryleneimide chromophores at the rim have been prepared using a novel synthetic approach. ${ }^{9}$ Within the research group, these molecules have been chosen for single molecule spectroscopy, ${ }^{10,11}$ because they allow topographic and optical observation to be combined on a single molecule level. To understand the dynamics of single molecule spectroscopy, it is essential to evaluate the photophysical processes at the ensemble level. Furthermore, these dendrimers have a high shape persistence, and through synthesis ${ }^{11}$ one can control the number of the peryleneimide chromophores.

In a separate publication, ${ }^{12}$ a comparative study of three generations of the polyphenylene dendrimers and a model compound using single photon timing will be reported. It was found that all dendrimers show a three exponential decay with similar decay times of the order of $350 \mathrm{ps}, 3-4 \mathrm{~ns}$, and 7-8 ns, while the model compound showed a monoexponential decay with a time constant of $4 \mathrm{~ns}$. The longest decay time of the dendrimers was attributed to a decay of an excimer-like entity. Using fluorescence upconversion ${ }^{13}$ and anisotropy transient absorption measurements on the second generation of this dendrimer family, ${ }^{14}$ an additional faster decay time of 5 ps was found. It was shown that upon excitation with $500 \mathrm{~nm}$ light a

* To whom correspondence should be addressed. Fax: +32 16327989 . E-mail: Frans.DeSchryver@chem.kuleuven.ac.be. locally excited state was formed prior to the formation of the complex. The dendrimer was further characterized by a high positive amplitude of the fast fluorescence decay component in the wavelength range between 560 and $600 \mathrm{~nm}$, while the model compound was found to decay in this wavelength range only on a nanosecond time scale. It was, thus, suggested that this 5 ps decay time is associated with the evolution into an excited state, which is delocalized between two or more chromophores in an excimer-like complex. This suggestion was verified for the second generation dendrimer by the observation of a growing in component upon excitation at $500 \mathrm{~nm}$ and detection at $620 \mathrm{~nm} .{ }^{13}$ Nevertheless, the question if and to what extent this complex is already preformed in the ground state was not answered.

In the present contribution, femtosecond time-resolved fluorescence excitation spectroscopy is used to study the correlation between the excited state dynamics and the ground state configuration of a multichromophoric dendrimer. In this approach, the detection wavelength is kept constant, while the wavelength used for the excitation is systematically tuned. This methodology was first reported by Glasbeek et al. ${ }^{15}$ In analogy to steady state excitation conditions, where this kind of spectroscopy is well established, applying this together with femtosecond time resolution yields additional and more detailed information about the correlation between the ground state configuration and the excited state kinetics. The third generation of the polyphenylene core dendrimer decorated with 16 peryleneimide chromophores at the rim (1, Scheme 1$)$ is compared to a model compound (2) in which a hexaphenylbenzene unit is attached to a peryleneimide. Furthermore, the setup that combines an optical parametric amplifier (OPA) as the excitation source and fluorescence upconversion as detection is described in detail. 
SCHEME 1: Molecular Structure of the Third Generation Polyphenylene Dendrimer 1 and the Model Compound Hexaphenylbenzene Peryleneimide 2
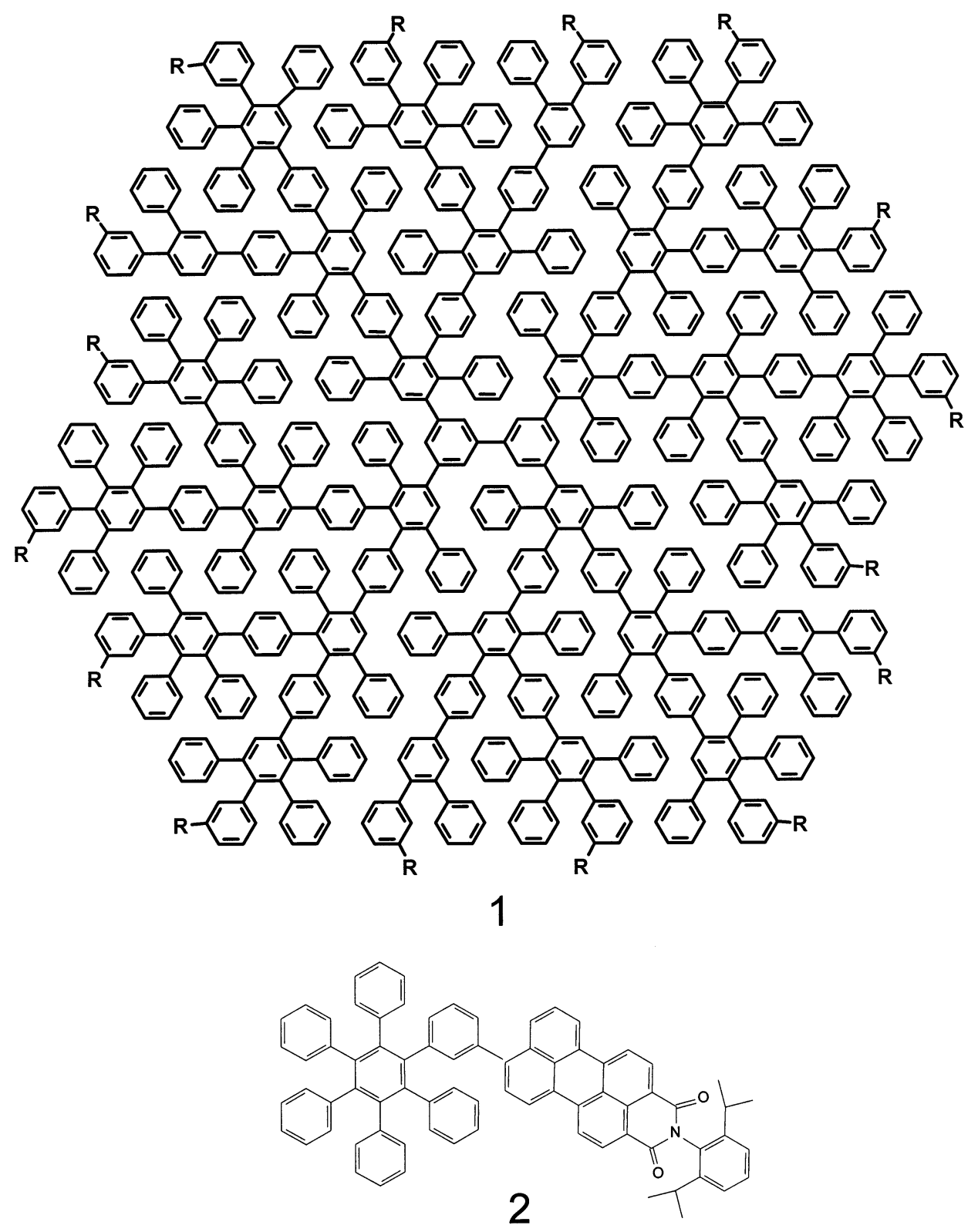

\section{Experimental Section}

The laser source has previously been described in detail. ${ }^{16}$ In brief, a Nd: $\mathrm{YVO}_{4}$ laser (Millennia V, Spectra Physics) is used to pump a Ti:sapphire laser (Tsunami, Spectra Physics). Its output seeds a regenerative amplifier (RGA, Spitfire, Spectra Physics). The output of the RGA (1 mJ, $100 \mathrm{fs}, 800 \mathrm{~nm})$ is split in two equal parts, one of which is used to pump an optical parametric generator/amplifier (OPA-800, Spectra Physics). The output wavelength range of the OPA is extended by harmonic generation using one or two $\mathrm{BBO}$ crystals, thus making a range of 300-900 $\mathrm{nm}$ accessible.

The experimental setup is shown in detail in Figure 1. The output of the OPA is passed through a multichromatic half wave plate (Berek compensator, New Focus 5540) and focused onto the sample using a $200 \mathrm{~mm}$ lens. The fluorescence is then collected by means of a $10 \times$ microscope objective lens (M10x, Newport) and focused by a $150 \mathrm{~mm}$ lens into an appropriate crystal with nonlinear optical properties. The cross correlation measured between the excitation and gate pulses had a width of $180 \mathrm{fs}$ (full width at half-maximum, fwhm). Besides this, the overall temporal resolution of our setup is limited by the angle $\alpha$ (refer to Figure 1) between the excitation beam and the collected fluorescence light. Using an angle of $10^{\circ}$, a temporal resolution of $260 \mathrm{fs}$ at a wavelength of $500 \mathrm{~nm}$ could be achieved.

The second part of the RGA output is sent into a variable delay line and focused by a $750 \mathrm{~mm}$ lens, the focal plane of which was selected to be $100 \mathrm{~mm}$ behind the nonlinear crystal (LBO, $1 \mathrm{~mm}$ ). The angle $\beta$ between this gate beam and the collected fluorescence was set to $7^{\circ}$, which was selected in order to allow for easy spatial filtering of the upconversion signal while still keeping a high conversion efficiency and good temporal resolution.

The generated sum frequency light was then collimated (300 $\mathrm{mm})$ and focused $(150 \mathrm{~mm})$ using UV-coated fused silica lenses (Newport) into the entrance slit of a $300 \mathrm{~mm}$ focal length monochromator (Acton Research Corp. 300-I, 600 lines/mm gratings blazed at 300 and $500 \mathrm{~nm}$ ). The signal passing through the output slit was detected by a UV-sensitive photomultiplier 


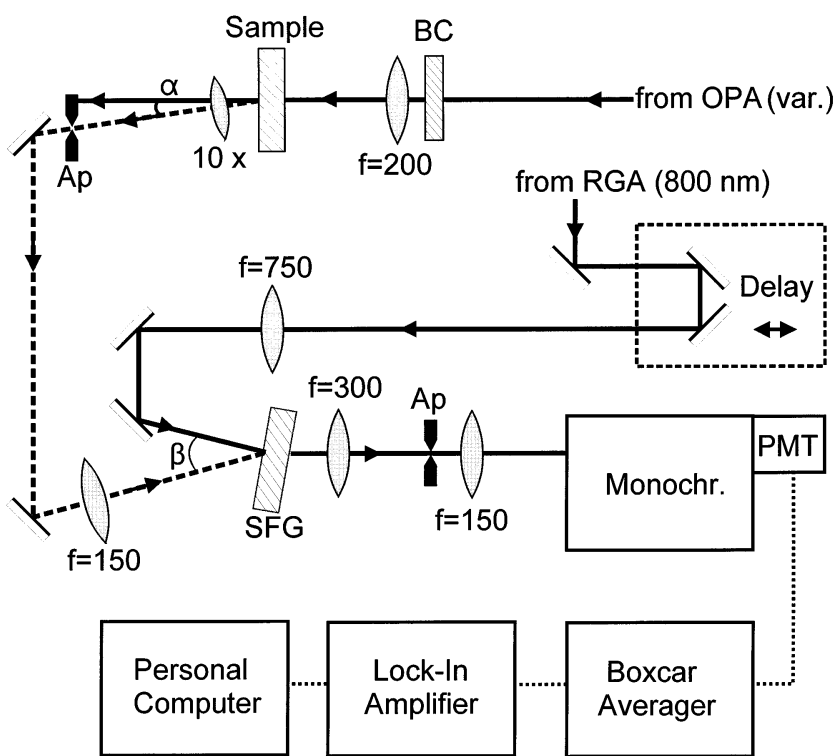

Figure 1. Experimental setup (schematic): OPA, optical parametric amplifier; AP, aperture; SFG, nonlinear optical crystal for sum frequency generation; BC, Berek Compensator; PMT, photomultiplier tube.

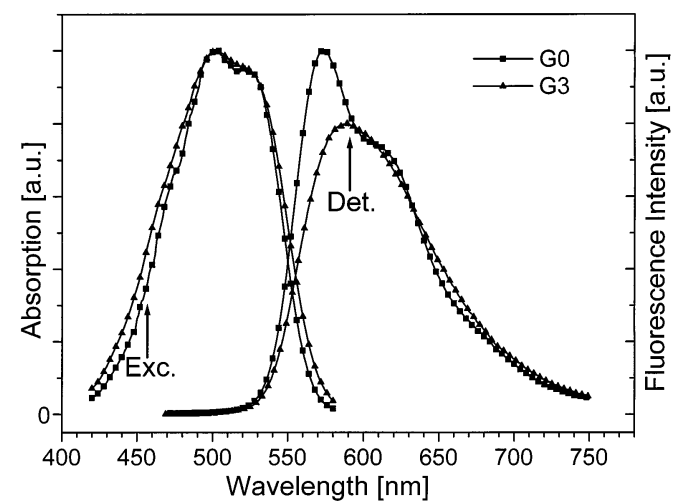

Figure 2. Excitation and emission spectra of $\mathbf{1}(\mathbf{\Delta})$ and $\mathbf{2}(\mathbf{\square})$ in chloroform at room temperature. Excitation spectra were detected at $590 \mathrm{~nm}$, fluorescence spectra were excited at $460 \mathrm{~nm}$.

tube (R1527p, Hamamatsu). The electrical signal from the photomultiplier tube was gated by a boxcar averager (SR 250, Stanford Research Systems), and the phase sensitivity was detected by a lock-in amplifier (SR 830, Stanford Research Systems). This scheme proved to be essential for the detection of fluorescence upconversion signals up to and at $700 \mathrm{~nm}$, as at those wavelengths various nonlinear processes generate a significant amount of additional light. The temporal response function of the system is shown (IRF) in Figure 3, which was determined by measuring the upconversion of light scattered from the sample.

\section{Results and Discussion}

The steady-state excitation and fluorescence spectra of $\mathbf{1}$ and 2 in chloroform at room temperature are shown in Figure 2. The excitation spectrum of $\mathbf{1}$ is similar to that of the model compound. The fluorescence spectra show more drastic differences; the spectrum of 2 has a maximum at $574 \mathrm{~nm}$ and a shoulder at $600 \mathrm{~nm}$, while the spectrum of $\mathbf{1}$ is structureless and its maximum is shifted to the red by $1450 \mathrm{~cm}^{-1}$. The excitation and fluorescence spectra as well as the quantum yield

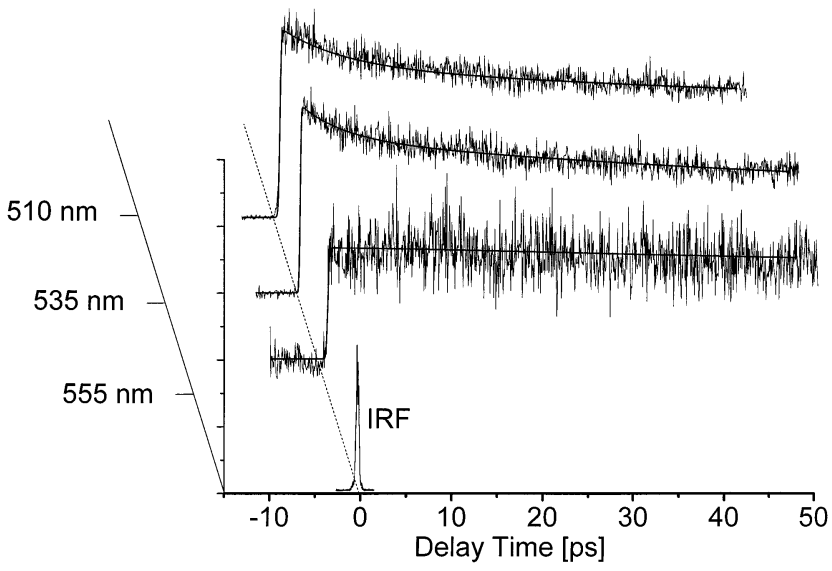

Figure 3. Fluorescence decay curves of 1 . The detection of the fluorescence was at $590 \mathrm{~nm}$, while the excitation was made at 510 , 535 , and $555 \mathrm{~nm}$. The solid lines are the corresponding fits, and IRF is the system prompt response.

of the third generation of the dendrimer 1 are identical to that of the second generation, which was reported previously. ${ }^{14}$

Upconversion measurements of the fluorescence decay were taken in two time windows of 50 and 420 ps. Figure 3 shows fluorescence upconversion decay curves of 1 which were excited at various wavelengths along with the corresponding fits. The detection wavelength was set at $590 \mathrm{~nm}$ in all cases.

The fluorescence decay curves of the dendrimer $\mathbf{1}$ and the model compound $\mathbf{2}$ could be fitted by a function that was the result of convolution of the instrument response function with sets of exponentials decay function of the form

$$
\Phi(t, \lambda)=\sum_{i} A_{i}(\lambda) \exp \left(-t / \tau_{i}\right)
$$

The fluorescence of $\mathbf{2}$ at $590 \mathrm{~nm}$ decays monoexponentially on a nanosecond time scale as reported previously. ${ }^{13,14}$ The data obtained for the dendrimer 1 could be fitted using a sum of three exponentials resulting in decay times of $\tau_{1}=5 \mathrm{ps}, \tau_{2}=$ $100 \mathrm{ps}$, and a few nanoseconds; the latter could not be determined exactly using upconversion in view of the data window limitation to $420 \mathrm{ps}$. The relative contribution to the total amplitude of the picosecond and nanosecond component at $535 \mathrm{~nm}$ were 0.4 and 0.6 , respectively. A similar distribution was found in $\mathrm{SPC}^{12}$ for the picosecond and nanosecond components. From previously measured SPT data, it is known that the decay on the nanosecond time scale is biexponential, with a longer decay component that was attributed to a decay of an excimer-like entity. ${ }^{12}$ Decay times of the same order of magnitude as $\tau_{1}$ and $\tau_{2}$ were found in fluorescence decay measurements of the second generation dendrimer. ${ }^{13}$ The values obtained for the third generation are tentatively attributed to similar processes. In particular, it is assumed that the fast decay component is related to the evolution from a locally excited chromophore to an excimer-like complex. ${ }^{13}$ In Figure 4 , the normalized relative amplitudes $A_{i}^{\mathrm{r}}=A_{i} / \sum_{i} A_{i}$ are depicted as a function of the excitation wavelength. The values for $A_{1}^{\mathrm{r}}$ and $A_{3}^{\mathrm{r}}$ are almost constant in the excitation wavelength range between 465 and $525 \mathrm{~nm}$, but at longer excitation wavelengths $A_{1}^{\mathrm{r}}$ decreases, becoming zero at $555 \mathrm{~nm}$, while $A_{3}^{\mathrm{r}}$ increases correspondingly. The relative amplitudes that are associated with the 100 ps decay component are almost constant over the whole excitation range. From SPT measurements reported separately, ${ }^{12}$ it is known that upon excitation of 1 at $560 \mathrm{~nm}$ the relative amplitude of the long decay component that was attributed to 


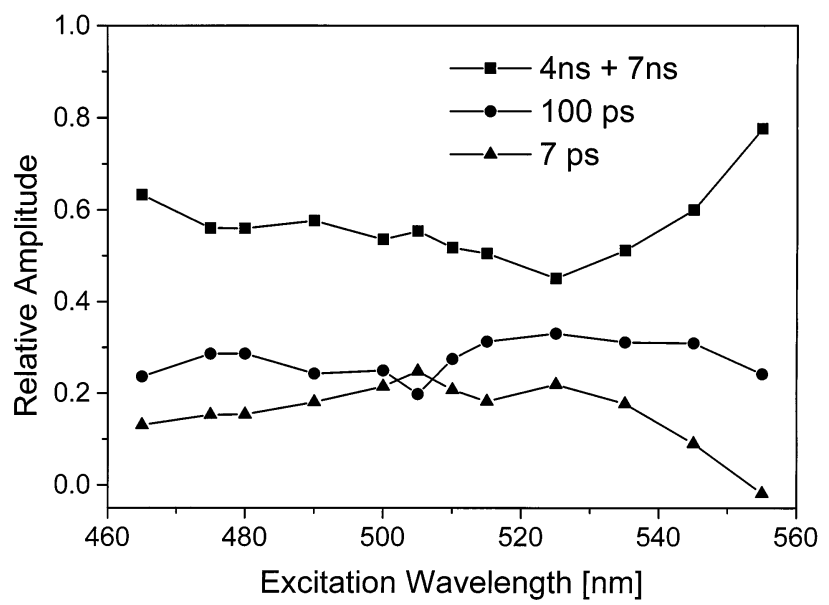

Figure 4. Relative amplitudes of the 5 ps (triangles), 100 ps (circles), and nanoseconds (squares) decay components plotted as a function of the excitation wavelength, measured in chloroform at room temperature.

a decay of a complex is the largest. Therefore, the correlation between the increase of the relative amplitude $A_{3}^{\mathrm{r}}$ and the decrease of the relative amplitude $A_{1}^{\mathrm{r}}$ substantiates the assumption that this component is related to the formation of an excimer-like complex in the excited state. Moreover, it can be deduced that at longer wavelengths the excitation is preferentially directly into the complex, which exists already in the ground state, while at shorter wavelengths excitation initially results to a large extent in a locally excited chromophore. Similar results were found in upconversion fluorescence measurements, where at longer detection wavelengths an increase of the amplitude of the long component was also observed. ${ }^{13}$

\section{Conclusions}

A new setup that combines the possibility of femtosecond excitation over the wavelength range from 300 to $900 \mathrm{~nm}$ with fluorescence upconversion detection was introduced. In particular, this combination allows time-resolved femtosecond excitation spectroscopy, which gives new sets of information on the correlation between the ground state conformation and the excited state processes in a multichromophoric dendrimer. It was found that upon irradiation at shorter wavelengths also a locally excited state is formed initially, which then evolves into an excited state complex. Excitation at longer wavelengths preferentially yields direct formation of an excited complex.

Acknowledgment. The authors gratefully acknowledge the FWO, the Flemish Ministry of Education for the support through GOA/1/96, the EC through the TMR Sisitomas, the Volkswagen-Stiftung, and the support of DWTC (Belgium) through IUAP-IV-11. J.H. thanks the FWO for a postdoctoral fellowship.

\section{References and Notes}

(1) Gopidas, K. R.; Leheny, A. R.; Caminati, G.; Turro, N. J.; Tomalia, D. A. J. Am. Chem. Soc. 1991, 113, 7335 10783

(2) Duan, R.; Miller, L.; Tomalia, D. A. J. Am. Chem. Soc. 1995, 117,

(3) Hawker, C. J.; Frechét, J. M. In Step-growth polymers for high performance materials: new synthetic methods; Hendrick, J. L., Labadie, J. W., Eds.; American Chemical Society: Washington, DC, 1996; p 132.

(4) Aoi, K. A.; Itoh, K.; Okada, M. Macromolecules 1995, 28, 5391.

(5) Archut, A.; Vögtle, F. Chem. Soc. Rev. 1998, 27, 233.

(6) Zimmerman, S. C.; Zeng, F. W.; Reichert, D. E. C.; Kolotuchin, S. V. Science 1996, 271, 1095 .

(7) Frechét, J. M. Science 1994, 263, 1710.

(8) Tomalia, D. A. Top. Curr. Chem. 1993, 165, 193. 1207.

(9) Morgenroth, F.; Kübel, C.; Müllen, K. J. Mater. Chem. 1997, 7,

(10) Hofkens, J.; Verheijen, W.; Shukla, R.; Dehaen, W.; De Schryver, F. C. Macromolecules 1998, 31, 4493.

(11) Gensch, T.; Hofkens, J.; Herrmann, A.; Tsuda, K.; Verheijen, W.; Vosch, T.; Christ, T.; Basche, T.; Müllen, K.; De Schryver, F. C. Angew. Chem., in press.

(12) To be published.

(13) Karni, Y.; Jordens, S.; De Belder, G.; Schweitzer, G.; Hofkens, J.; Gensch, T.; Maus, M.; De Schryver, F. C.; Herrmann, A.; Müllen, K. Chem. Phys. Lett. 1999, 310, 73

(14) Hofkens, J.; Latterini, L.; De Belder, G.; Gensch, T.; Maus, M.; Vosch, T.; Karni, Y.; Schweitzer, G.; De Schryver, F. C.; Herrmann, A.; Müllen, K. Chem. Phys. Lett. 1999, 304, 1.

(15) Marks, D.; Prosposito, P.; Zhang, H.; Glasbeek, M. Chem. Phys. Lett. 1998, 289, 535.

(16) Schweitzer, G.; Xu, L.; Craig, B.; De Schryver, F. C. Opt. Commun. 1997, 142, 283. 